\title{
ENGLISH VERSION: PERSONALIZED DESENSITIZATION WITH ACETYLSALICYLIC ACID IN PATIENTS WITH HYPERSENSITIVITY TO NON- STEROIDAL ANTI-INFLAMMATORY DRUGS
}

\author{
Lavrenko A.V., Avramenko Ya.M., Borzykh O.A., Kaidashev I.P. \\ Ukrainian Medical Stomatological Academy
}

Aims: Hypersensitivity to nonsteroidal anti-inflammatory drugs (NSAIDs) has various mechanisms and represents different clinical syndromes from anaphylaxis to severe bronchospasm. The prevalence of aspirin hypersensitivity among patients with asthma and nasal polyps reaches $25.6 \%$. Respiratory reactions associated with aspirin or other NSAIDs are not immunological. The basis of these reactions is non-allergic hypersensitivity of the cross-reactive type. Desensitization followed by long-term aspirin therapy is an effective method of treating hypersensitivity to aspirin or other NSAIDs. Using aspirin 600-1200 mg/day can significantly alleviate the symptoms of asthma, allergic rhinitis. Methods: We successfully applied aspirin desensitization for method of patients with hypersensitivity to NSAIDs. According to the method, an hour before the desensitization, daily montelukast $10 \mathrm{mg}$ was taken orally, then aspirin every 3 hours. Results: Three patients underwent desensitization of aspirin. The dose was selected individualy depending on the clinical manifestations of drug-induced adverse reactions (AR). ARs during desensitization were treated by iv dexamethasone administration. Subsequent doses did not cause AR. Doses of aspirin were increased to a maximum of $1250 \mathrm{mg}$ daily, and were continued for the long-term use. Conclusion: It is possible to conclude that the initial dose of aspirin should be 16-40mg; it is possible to increase the dose if the initial dosage is well tolerated; symptoms of moderate intolerance are treated by 4-8 mg iv dexamethasone; prior to desensitization, we recommended to use montelukast $10 \mathrm{mg}$, it is safe to practice desensitization of aspirin according to a personalized technique by a specialist in an intensive care unit.

Keywords: aspirin desensitization, asthma, personalized treatment.

After the first presentation of hypersensitivity reaction to aspirin (ASA) by Hirschberg in 1902, several types of hypersensitivity reactions to non-steroidal antiinflammatory and anti-rheumatic drugs (NSAIDs) have been described. NSAID-induced hypersensitivity reactions have different mechanisms and represent a wide range of clinical manifestations, from anaphylaxis to severe bronchospasm, developing within minutes after administration of drugs, as well as delayed reactions, developing in several days or weeks [1].

Hypersensitivity to ASA is found in $0.5-1.9 \%$ of population. The prevalence of hypersensitivity to NSAIDs in asthma patients as reported by questionnaire survey or medical record evaluation is from $4.3 \%$ to $11 \%$ and may reach $21 \%$ if provocative tests are used to clarify the diagnosis. Among patients with bronchial asthma and nasal polyps, the prevalence of hypersensitivity to ASA may be as high as $25.6 \%$. Skin reactions to NSAIDs occur in $3.5 \%$ of population, and the prevalence of hypersensitivity to ASA can reach $27-35 \%$ among patients with chronic urticaria. NSAIDs constitute the second most frequent group of drugs responsible for the development of anaphylactic reactions [2].

Allergic reactions to NSAIDs are observed regardless of their chemical structure and / or anti-inflammatory potential. NSAIDs from the heteroaryl acetic acid group (naproxen, diclofenac, ibuprofen) cause immediate hypersensitivity reactions more frequently than other NSAIDs. Modern selective cyclooxygenase-2 inhibitors can also cause allergic reactions, although with a very low frequency of $0.008 \%$.
Hypersensitivity reactions caused by ASA or other NSAIDs (synonyms, used previously, are "aspirin triad" and "asthmatic triad") are manifested by bronchial obstruction, shortness of breath, nasal congestion or rhinorrhea, and are observed in patients with chronic respiratory diseases (asthma, rhinosinusitis and nasal polyps).

The prevalence of this group of reactions ranges from 4.3 to $20 \%$, depending on the population of the examined subjects and diagnostic methods. The presence of chronic rhinosinusitis with nasal polyps, severe asthma and female sex are associated with a high incidence of hypersensitivity to ASA and other NSAIDs.

Respiratory system responses, caused by ASA or other NSAIDs, are not immunological, i.e. they are not based on an antigen-antibody interaction. The nonallergic hypersensitivity of the cross-reactive type underlies these reactions. The main mechanism of these reactions consists in the inhibition of COX-1 by most NSAIDs - COX-1 inhibitors. Such a change in COX-1 activity is clearly manifested under conditions of impaired arachidonic acid metabolism. The disorders of arachidonate metabolism are as follows: 1) increased levels of leukotriene E4 in urine, expired breath condensate, bronchoalveolar lavage, induced phlegm and saliva due to increased 5-lipoxygenase activity; 2) decreased production of prostaglandin E2 by the epithelium of the upper and lower respiratory tract, accompanied by the inhibition of COX-2 and reduced basal production of lipoxin A4 by peripheral blood leukocytes; 3) increased expression of type 1 leukotriene receptors by the nasal mucosa cells [3].

To cite this English version: A.V. Lavrenko, Ya.M. Avramenko, O.A. Borzykh, I.P. Kaidashev Personalized desensitization with acetylsalicylic acid in patients with hypersensitivity to non-steroidal anti-inflammatory drugs// The Medical and ecological problems. 2020. - Vol 24, № 1-2. - P. 40-43. 
Inhibition of COX-1 leads to activation of mast cells and eosinophils with a release of inflammatory mediators. Therefore, such reactions are characterized by manifestations of chronic eosinophilic inflammation. COX-2 inhibitors and low doses of COX-1 inhibitors are usually well tolerated by patients. Cysteinyl-leukotrienes (LT) are one of the main mediators, causing NSAID intolerance reactions. Consequently, blockade of type 1 leukotriene receptors by pharmacological inhibitors can alleviate clinical symptoms.

Viral infections may be an important factor in the persistence of respiratory tract inflammation in such patients.

Genetic polymorphism may play a certain role in the development of hypersensitivity reactions to NSAIDs. Associations of respiratory diseases, exacerbated by NSAIDs intake, with dipeptidyl peptidase-10 polymorphism, ALOX-15, IL-5 receptor, COX-1, etc. have been described $[4,5]$.

Clinical manifestations of hypersensitivity as a rule are observed in 30-180 min after administration of ASA / NSAIDs: patients develop non-bronchial symptoms (rhinorrhea, nasal congestion), ocular, skin (urticaria and / or angioedema) and gastrointestinal symptoms. Usually, a patient with hypersensitivity to NSAIDs presents with past history of chronic rhinosinusitis (sometimes complicated by mucosal hypertrophy and nasal polyps) and / or asthma. However, in some patients the intake of NSAIDs may cause the first bronchial asthma attack. Anosmia is a characteristic clinical feature in such patients.

Desensitization, followed by a long-term ASA therapy, is an effective treatment for patients with respiratory diseases, exacerbated by NSAIDs. The use of ASA at a dose of $600-1200 \mathrm{mg}$ per day can significantly alleviate the symptoms of asthma and affected upper respiratory tract, as well as reduce the dose of intranasal corticosteroids (recommendation level B). In some patients, desensitization with ASA leads to a decreased frequency of polyps recurrence and repeated surgeries. Along with improving the management of bronchial asthma, desensitization with ASA also reduces the need for oral glucocorticoids [6].

We have tested and successfully applied the technique of desensitization with acetylsalicylic acid for patients with hypersensitivity to NSAIDs. One hour prior to desensitization, patients were prescribed montelukast 10 mg orally, 1 tablet, and ASA every 3 hours.

We present several typical cases that demonstrate an individual approach to desensitization.

\section{Clinical case No. 1}

Patient N., 25 years old, presented with complaints of low-productive cough with white sputum; cough persisted throughout the day; nasal congestion, hypernasal voice, lack of sense of smell. Case history: these symptoms had persisted since August 2017, starting with cough and nasal congestion. The patient had been treated by otolaryngologist for sinusitis. In November 2017, the patient had sustained an injury (back muscles strain) in a gym; she had been prescribed diclofenac sodium. After injection of the drug, the patient had developed bronchospasm for the first time. The attack had been arrested by a team of emergency medical service with dexamethasone $4 \mathrm{mg}$ i/v by stream infusion.

For the second time, bronchospasm attack had developed on taking 2 tablets of ibuprofen for menstrual pain. The patient had arrested the attack by oral admini- stration of cetirizine hydrochloride $10 \mathrm{mg}$ and dexamethasone $4 \mathrm{mg} \mathrm{i} / \mathrm{m}$.

The patient referred to allergist who prescribed a checkup, during which an increase in IgE (172 IU / ml) and changes in the spirogram were detected. Before desensitization, the patient was examined:

Blood test for total IgE (31.01.2018) - $172 \mathrm{IU} / \mathrm{ml}$ (norm is up to 100.0).

07.02.2018: Allergic tests. Pollen screening panel. Allergen from the pollen of birch, willow, black alder, hazel, poplar, dandelion, plantain, timothy grass, cocksfoot, couch grass, common ragweed, common wormwood, meadow fescue, rye, grass, trees - the results were negative. Household panel: allergen from cat and dog dander, pillow feathers, the mixture of $D$. pteronyssius and farinae ticks - the results were negative. Conclusion: No sensitization to pollen, household, epidermal allergens was detected.

08.07.2018: X-ray of thoracic organs. Conclusion: No radiographically visible focal and infiltrative changes in the lungs were detected.

09.07.2018: Consultation of otolaryngologist. Diagnosis: Allergic rhinosinusopathy.

09.07.2018: Allergic tests were repeated. Pollen screening panel. Allergen from the pollen of birch, willow, black alder, hazel, poplar, dandelion, plantain, timothy grass, cocksfoot, couch grass, common ragweed, common wormwood, meadow fescue, rye, grass, trees - the results were negative.

09.07.2018: Complete blood count from the vein: increased eosinophils - 9\% (norm is up to $0.5-5.5 \%$ ), no other deviations from the norm.

17.10.2018: Multispiral computed tomography of the paranasal sinuses. Conclusion: Spiral computed tomography pattern of hyperplastic pansinusitis.

Nasocytogram (22.10.2018): increased neutrophil granulocytes - $87 \%$ (norm is up to $65-75 \%$ ); eosinophilic granulocytes $-8 \%$ (norm is up to $0-5 \%$ ), no other deviations from the norm.

24.10.18: Clinical diagnosis: Polypous rhinosinusitis, intolerance to non-steroidal anti-inflammatory drugs (NSAIDs). Compensated BA.

The following treatment was prescribed: cetirizine hydrochloride $10 \mathrm{mg} 1 \mathrm{tab}$ once a day, budesonide / formoterol 160 / 4.5 pg 1 dose once a day, montelukast sodium $10 \mathrm{mg} 1$ tab once a day; consultation with otolaryngologist.

After examination, the patient was recommended to undergo desensitization to NSAIDs.

\section{Conduction of desensitization:}

The 1st dose of ASA was taken orally on 13.11.18 $16 \mathrm{mg}$, the 2nd dose was $37.5 \mathrm{mg}, 30$ minutes after receiving the second dose, the patient developed nasal congestion, dry wheezing, shortness of breath, bronchospasm, which were arrested with dexamethasone $4 \mathrm{mg}$ i/v by stream infusion; after arresting bronchospasm, the 3rd dose of ASA continued - $75 \mathrm{mg}$; in 30 minutes the patient again developed bronchospasm with rhinorrhea, dry wheezing, shortness of breath, which were arrested with dexamethasone $8 \mathrm{mg}$ i/ $\mathrm{v}$ by stream infusion and inhalation of salbutamol $100 \mu \mathrm{g}, 1$ dose. On this day, ASA intake at a dose of $75 \mathrm{mg}$ was discontinued.

The next day, on 14.11.18, one hour prior to administration of ASA, the patient took montelukast orally 10 $\mathrm{mg}, 1 \mathrm{tab}$, then the 1st dose of ASA (37.5 mg) was administered; the 2nd dose was $75 \mathrm{mg}$; the patient did not present any complaints, the 3rd dose was $150 \mathrm{mg}$; the 
patient did not present any complaints; at a dose of 150 mg the drug intake was discontinued.

15.11.18: one hour prior to administration of ASA, the patient took montelukast orally $10 \mathrm{mg}, 1 \mathrm{tab}$, then the $1 \mathrm{st}$ dose of ASA (225 mg) was administered; the 2nd dose was $300 \mathrm{mg}$; the $3 \mathrm{rd}$ dose was $375 \mathrm{mg}$; the patient did not present any complaints on that day.

16.11.18: One hour prior to administration of ASA, the patient took montelukast orally $10 \mathrm{mg}, 1$ tab; the 1 st dose of ASA was $625 \mathrm{mg}$; the 2nd dose - $625 \mathrm{mg}$; the patient did not present any complaints. After reaching the maximum dose of ASA, the patient was discharged from the department with appropriate recommendations.

\section{Clinical case No. 2}

Patient M., 39 years old, presented with complaints of nasal congestion, rhinorrhea, hypernasal voice, lack of sense of smell, constant intake of vasoconstrictor drugs. Case history: the patient had been ill since 2011, when she had noticed the annoying cough and nasal congestion. She had referred to allergist; assays for worm infestation and allergy tests had been prescribed. The results had been normal. In 2011, during menstruation, the patient had taken ketorolac trometamol $30 \mathrm{mg}$ for the first time. In 30 minutes, the patient had developed bronchospasm (wheezing, bursting chest pain, facial hyperemia). The patient had arrested the attack with dexamethasone and chloropyramine hydrochloride $25 \mathrm{mg}$.

The second episode of bronchospasm had occurred in the patient upon taking 1 tablet of ASA during SARS; the clinical presentation of bronchospasm was the same as when taking ketorolac. The attack had been arrested by the patient with dexamethasone $4 \mathrm{mg} \mathrm{i} / \mathrm{m}$ and chloropyramine $25 \mathrm{mg} 1 \mathrm{tab}$. The patient was examined:

09.11.2011: Analysis for total lgE - 86.7 IU / ml (norm is up to 87.0); Ab to Ascaris IgG - 0.17 (norm is up to 0.17); Ab to Trichinella lgG - 0.16 (norm is up to 0.16); IgE to allergens of dust Dermatophagoides pteronyssinus, Dermatophagoides farina, German cockroach $<0.1$ $\mathrm{kU} / 1$ were absent, IgE to allergens of microorganisms of Penic notatum, Cladospor herbarum, Asper fumigatus, Can albicans, Altenaria tenuis group $<0.1 \mathrm{kU} / 1$ were absent.

15.12.2011: Spirogram: Moderate bronchial asthma by obstructive type. Test with bronchial spasmolytic (salbutamol $300 \mu \mathrm{g})$ - the result was positive. FEV1 Act1 2.19; \% (A1 / P ) - 67.3\%; Act2 - 2.58; \% (A2 / P) $79.5 \%$; \% (A2 / A1) - 118.0\%.

13.01.16: MRI (the paranasal sinuses). Conclusion: CT signs of polypous rhinosinusitis.

Diagnosis: Polyposis of the nasal sinuses. The patient refused to undergo the recommended surgical treatment. Hormonal treatment with Avamis nasally and desensitization with acetylsalicylic acid were recommended.

\section{Conduction of desensitization:}

The 1st dose was taken orally on 23.10 .18 - $18 \mathrm{mg}$; the patient did not present any complaints; the 2nd dose was $37 \mathrm{mg}$; the patient did not present any complaints; the $3 \mathrm{rd}$ dose - 75mg; 30 minutes after receiving the $3 \mathrm{rd}$ dose, the patient developed nasal congestion, rhinorrhea, sneezing, difficulty breathing; these symptoms were arrested with $4 \mathrm{mg}$ of dexamethasone i/ $\mathrm{v}$ by stream infusion; the 4th dose was 150 mg; the patient did not present any complaints.

The next day, 24.10.18, the 1st dose of ASA was 225 $\mathrm{mg}$, the 2nd dose was $300 \mathrm{mg}$, the $3 \mathrm{rd}$ dose was $375 \mathrm{mg}$, and the 4th dose was $450 \mathrm{mg}$.
25.10.18: on the last day of desensitization, ASA at a dose of $500 \mathrm{mg}$ was administered: the 1st dose of ASA was $625 \mathrm{mg}$; the 2nd dose - $625 \mathrm{mg}$; the patient did not present any complaints. The patient was discharged from the department with a maximum dose of ASA (1250 mg).

\section{Clinical case No. 3}

Patient I., 28 years old, presented with complaints of nasal congestion, hypernasal voice, rhinorrhea, lack of sense of smell. Case history: these symptoms had appeared since 2006. The patient had applied selftreatment with vasoconstrictor drops. In 2012, the condition had been aggravated with cough, shortness of breath, and the following diagnosis had been made: Bronchial asthma. Polypous rhinosinusitis. Deflection of the nasal septum. In 2014, the patient underwent surgery for polyps and deflected nasal septum.

In 2015, during the menstrual cycle, the patient had taken a pill of ketorolac. She had developed bronchospasm, which had been manifested by dry cough, sore throat, suffocation. The patient was examined:

02.03.2016: Allergic tests, ISAC package - the result was negative.

08.07.2014: Complete blood count: $R B C-4.58 * 10^{12}$ / l; Hb - $140 \mathrm{~g} / \mathrm{l} ; \mathrm{WBC}-5.97 * 10^{9} / \mathrm{l} ; \mathrm{ESR}-4 \mathrm{~mm} / \mathrm{h}$; $P L-322 * 10^{9} /$ l; leucogram: e. $-23.1 \%$; bas. $-2.3 \%$; s. - 37.9\%; lymph. - 29.8\%; mon. - 6.9\%. Blood glucose $4.96 \mathrm{mmol} / \mathrm{I}$

08.07.2014: Markers of viral hepatitis $B$ and $C$ were negative.

After examination, clinical diagnosis was made: Polypous rhinosinusitis, intolerance to non-steroidal antiinflammatory drugs (NSAIDs). Compensated BA.

After examination, the patient was recommended to undergo desensitization to NSAIDs.

\section{Conduction of desensitization:}

The 1st dose was taken orally on 19.06.18 - $40 \mathrm{mg}$; moderate rhinorrhea appeared; the 2nd dose - 40 mg; 30 minutes after the 2 nd dose, the patient developed nasal congestion, rhinorrhea, sneezing, sore throat and dry rales, which were arrested with $4 \mathrm{mg}$ of dexamethasone i/v by stream infusion; after arresting bronchospasm, the $3 \mathrm{rd}$ dose continued - $100 \mathrm{mg}$; the patient did not present any complaints.

The next day, on 20.06.18, the 1st dose of ASA was $125 \mathrm{mg}$, the patient did not present any complaints; the 2nd dose was $250 \mathrm{mg}$, the $3 \mathrm{rd}$ dose - $375 \mathrm{mg}$; the patient did not present any complaints on that day.

21.06.18: One hour prior to administration of ASA, the patient took montelukast $10 \mathrm{mg}$ orally (1 tab); on the last day of desensitization, ASA at a dose of $500 \mathrm{mg}$ was administered: the 1st dose of ASA was $625 \mathrm{mg}$; the 2nd dose $-625 \mathrm{mg}$. The patient did not present any complaints. The patient was discharged from the department with a maximum dose of ASA (1250 mg).

After desensitization and further administration of ASA, the patient's status was satisfactory. In August 2018, the patient developed rhinorrhea, nasal congestion. The patient was prescribed a check-up:

29.08.2018: Blood test for lgE - $258 \mathrm{IU} / \mathrm{ml}$ (norm is up to 20).

04.09.2018: Allergic tests. Pollen screening panel. There were increased concentrations of immunoglobulin $E$ to golden-rod - $0.894 \mathrm{kU} / \mathrm{I}$ (class II 0.70-3.49); to dandelion - $2.54 \mathrm{kU} / \mathrm{I}$ (class II 0.70-3.49); to wormwood $11.2 \mathrm{kU} / \mathrm{I}$ (class III $3.50-17.49$ ). 
Following the examination, it was found that the patient had sensitization with pollen antibodies. The patient was recommended to undergo specific immunotherapy with wormwood pollen.

\section{Discussion}

Three patients were desensitized with acetylsalicylic acid, and desensitization was personalized by nature. A dose of ASA was selected individually for each patient, depending on the anamnestic clinical manifestations of anaphylactic reactions. Two patients had an allergic reaction in the form of bronchospasm after ASA intake: one dose of $40 \mathrm{mg}$ of ASA and the other dose of $37.5 \mathrm{mg}$ of ASA. The 3rd patient developed rhinorrhea and sneezing on administering the 3rd dose of ASA $(75 \mathrm{mg})$. In all three patients, allergic reactions were arrested by $\mathrm{i} / \mathrm{v}$ administration of dexamethasone. Later on, subsequent doses did not cause allergic reactions. Doses of ASA were raised to a maximum of $1250 \mathrm{mg}$ per day in 2 doses for a long-term administration.

One patient subsequently presented with clinically significant sensitization to wormwood pollen. In our opinion, this sensitization was masked by severe intolerance to ASA and revealed a seasonal allergy after desensitization.

Thus:

1) The initial dose of ASA should be $16-40 \mathrm{mg}$, depending on the presence of severe reactions in case history;

2) An increase in the dose of ASA is performed in case of satisfactory dose tolerance (mild rhinorrhea symptoms are allowed);

3) The appearance of moderate intolerance symptoms to ASA is effectively arrested by $\mathrm{i} / \mathrm{v}$ administration of 4-8 $\mathrm{mg}$ of dexamethasone;
4) Most often, the symptoms of intolerance in desensitization with ASA are manifested when receiving doses of $37-75 \mathrm{mg}$;

5) It is advisable to administer $10 \mathrm{mg}$ of monteleukast before desensitization with ASA;

6) Manifestation of desensitization with ASA is a safe technology when conducted using a personalized technique by trained personnel under conditions of intensive care wards;

7) Successful desensitization with ASA can contribute to the detection of seasonal allergies, previously hidden by year-round symptoms.

\section{References}

1. Kowalski ML, Makowska JS. Seven steps to the diagnosis of NSAIDs hypersensitivity: how to apply a new classification in real practice? Allergy Asthma Immunol Res. 2015 Jul;7(4):312-20. doi: 10.4168/aair.2015.7.4.312.

2. Kowalski ML, Asero R, Bavbek S, Blanca M, Blanca-Lopez $\mathrm{N}$, Bochenek $\mathrm{G}$, et al. Classification and practical approach to the diagnosis and management of hypersensitivity to nonsteroidal anti-inflammatory drugs. Allergy. 2013 Oct;68(10):1219-32. doi: 10.1111/all.12260.

3. Picado C. Mechanism of aspirin sensitivity. Curr Allergy Asthma Rep. 2006 May;6(3):198-202.

4. Kim SH, Choi H, Yoon MG, Ye YM, Park HS Dipeptidylpeptidase 10 as a genetic biomarker for the aspirinexacerbated respiratory disease phenotype. Ann Allergy Asthma Immunol. 2015 Mar;114(3):208-13. doi: 10.1016/j.anai.2014.12.003..

5. Ledford DK, Wenzel SE, Lockey RF. Aspirin or other nonsteroidal inflammatory agent exacerbated asthma. J Allergy Clin Immunol Pract. 2014 Nov-Dec;2(6):653-7. doi: 10.1016/j.jaip.2014.09.009.

6. Chu DK, Lee DJ, Lee KM, Schünemann HJ, Szczeklik W, Lee JM. Benefits and harms of aspirin desensitization for aspirin-exacerbated respiratory disease: a systematic review and meta-analysis. Int Forum Allergy Rhinol. 2019 Sep 13. doi: 10.1002/alr.22428.

Матеріал надійшов до редакції 14.02.2020. 